\title{
Simulation Analysis of Microchannel Deformation during LTCC Warm Water Isostatic Pressing Process
}

\author{
Lang Ping, Zhaohua Wu* \\ Electromechanical Engineering College, Guilin University of Electronic Technology, Guilin, 541004, \\ China
}

Keywords: Low Temperature Co-fired Ceramic, Micro-channel deformation, simulation, Abaqus.

\begin{abstract}
During the process of warm water isostatic pressing process, the internal micro-channels fabricated in Low Temperature Co-fired Ceramic (LTCC) substrate are prone to collapse. In this paper, a three-dimensional model of LTCC microwave component is established by the finite element software ABAQUS, and the micro-channel deformation is studied by the simulation analysis of warm water isostatic pressing process. Results show that pressure is the main factor that leads to the deformation of micro-channel, and temperature is followed. At the same time, the microchannel with large width-height ratio-aspect ratio has large deformation. Choose smaller pressure $18 \mathrm{MPa}$ and high temperature $80{ }^{\circ} \mathrm{C}$, microchannel deformation can be controlled below $15 \%$.
\end{abstract}

\section{Introduction}

As the rapid development of modern technology, electronic product has expanded from traditional two-dimensional (2D) assembly to high density three-dimensional (3D) assembly. Low Temperature Co-fired Ceramic (LTCC) has become an ideal substrate material [1] for 3D high density assembly thanks to its perfect features: chemical resistance, hermeticity, low dielectric constant, matching of thermal expansion coefficient with the silicon and integration of passive components. Meanwhile micro-channels are easy to realize in the LTCC substrate, using liquid cooling, which can better solve the cooling problem brought by the highly integrated system. Warm water isostatic pressing with uniform pressure has become the most commonly used laminating process. The typical lamination parameters are $20 \mathrm{MPa}$ at $70^{\circ} \mathrm{C}$ for $10 \mathrm{~min}$. During lamination the green LTCC tape are properly joined together. However, the relative high pressure can usually result in microchannel deformation during warm water isostatic pressing [2]. To reduce the deformation of microchannel, sacrifice volume material (SVM) is used to fill the micro-channel during warm isostatic pressing process [3]. So far the study of the microchannel deformation of the LTCC isostatic pressing and optimization is mostly adopt the method of experimental research. In this paper, a three-dimensional model of LTCC microwave component is established by the finite element software ABAQUS. By simulation analysis of the microchannel deformation, the lamination parameters are optimized.

\section{Micro-channels forming and deformation}

The Micro-channel deformation in the manufacturing process is the main problems existing in the system manufacturing. General punch forming process is used firstly to form the 2D microchannel in a single green tape, and then the 3D microchannel can be formed after stacking, warm water isostatic pressing and sintering [4]. Warm water isostatic pressing, using the PASCAL's principle, the separate LTCC green tapes will finally join together under uniform pressure in every direction and the action of heat. LTCC green tape mainly contains of ceramic particles and glass material and organic material. During warm water isostatic pressing process, the rheological property of green tape increases as the lamination pressure rises. Micro-channel will collapse when lamination pressure exceed greatly [3]. The simplest model of green tape is of an elastic-plastic material [5]. Plastic deformation will produced when pressure exceeds the yield point of the green 
tape. With the increasing of temperature, the yield point decreases, therefore a lower lamination pressure is available. Considering of the time-varying deformation response of the LTCC green tape occurs due to the viscoelastic nature of the polymer system and the heat effect, viscoelastic constitutive model is more reasonable [6] for LTCC green tape during warm water isostatic pressing, especially in structures that contains micro-channels do not have isostatic pressure loading. In this paper, viscoelastic constitutive model will be used for LTCC green tape, and elastic-plastic constitutive model will be used for SVM.

In the finite element software ABAQUS generalized Prony model is used to describe the viscoelastic properties of material, generalized Prony model and generalized Maxwell model have the same mathematical description, which can better reflect the viscoelastic properties of material. The basic form of generalized Prony series can be given as follow:

$$
\begin{gathered}
G(t)=G_{\infty}+\sum_{i=1}^{n_{G}} G_{i} \exp \left(-\frac{t}{\tau_{i}^{G}}\right) \\
K(t)=K_{\infty}+\sum_{i=1}^{n_{K}} K_{i} \exp \left(-\frac{t}{\tau_{i}^{K}}\right)
\end{gathered}
$$

Where $G_{\infty}$ and $G_{i}$ are shear modulus, $K_{\infty}$ and $K_{i}$ are bulk modulus, $\tau_{i}^{G}$ and $\tau_{i}^{K}$ are the relaxation time of Prony series. In this paper, the Prony parameters of viscoelastic model can be obtained by using ABAQUS to fit the green tape stress relaxation curve.

\section{Structure of the LTCC microwave module}

LTCC microwave module is fabricated by 25 layers of Ferro LTCC green tape, each layer with the thickness of $0.127 \mathrm{~mm}, 40 \mathrm{~mm}$ in width and $40 \mathrm{~mm}$ in length. The cross section size of the two main channels is $2.20 \mathrm{~mm}$ in width and $0.762 \mathrm{~mm}$ in height, and the cross section size of the eight branch channels is $1.0 \mathrm{~mm}$ in width and $0.635 \mathrm{~mm}$ in height. The two main channels connect the eight branch channels, which forms a channel network. Polypropylene carbonate material is filled in the microchannels as sacrifice volume material. The size of the gap between SVM and the green tape is about $0.1 \mathrm{~mm}$ according to the actual production process. At the same time, this paper mainly study of micro-channel deformation and other structures within the components such as electrical wiring, metal column and so on has carried on the proper simplification, as shown in Figure 1. The entire model is divided by hexahedral C3D8T element, with the total of 41392 elements and 50869 nodes. The meshes of the microchannels are properly refined, as shown in Figure 2.

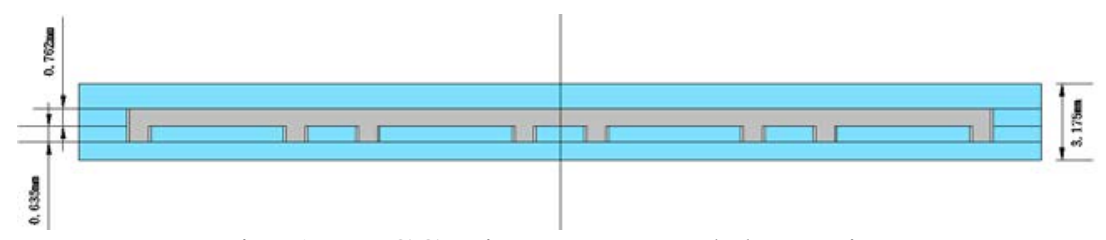

Fig. 1 LTCC microwave module section

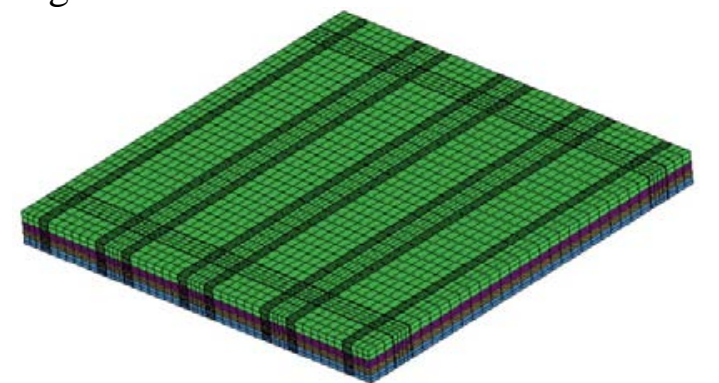

Fig. 2 LTCC microwave module element mesh

LTCC green tapes after stacking are put into the vacuum bag. The water is heated to the specified value, and then the pressure is loaded. Warm water isostatic pressing involves a combination of heat and force, belongs to the thermal structure coupling analysis. This paper adopts 
the method of direct thermal structure coupling of ABAQUS simulation. In the process, heat convection and uniformly pressure is loaded on the model outside surface. The convection coefficient is $300 \mathrm{~W} /(\mathrm{m} 2 \cdot \mathrm{K})$. The entire model is in the force equilibrium state, free body constraints are imposed on it.

The whole simulation process is divided into five steps. In the first step the green tapes are heated to the specified temperature, and in the second step uniformly pressure is loaded on the model, and in the third step pressure remains constant, in the fourth step pressure is unload, the last step the laminated green tapes are cooled to the room temperature.

\section{Simulation analysis}

\subsection{Typical warm water isostatic pressing process.}

The main lamination parameters of LTCC water isostatic pressing process are pressure, temperature and time. In this paper, the influence of the three main lamination parameters on microchannel deformation is studied by the finite element simulation analysis. By the influence law of main lamination parameters on the micro-channel deformation, the lamination parameters are optimized. The typical lamination parameters are $20 \mathrm{MPa}$ at $70^{\circ} \mathrm{C}$ for $10 \mathrm{~min}$, the micro-channel height direction deformation will be extracted.
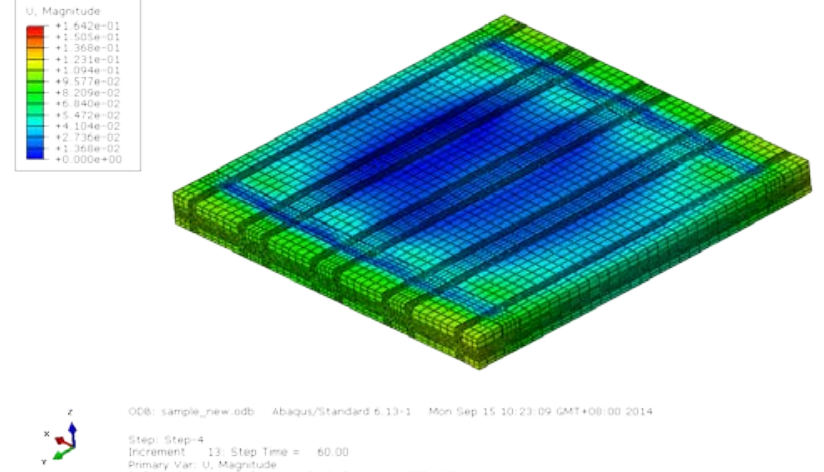

Fig. 3 distribution of the total displacement after unloading

The Figure 3 shows that overall shrinkage occurs after unloading, the micro-channel deformation is relatively large, the main channels and the branch channels happens to a certain degree of collapse. Result shows that isostatic pressing process can provide uniform pressure to make the component overall deformation, keep a certain roughness, but for the component that embedded micro-channel network suffers larger deformation due to uniform pressure [5]. The displacement of micro-channels cross-section on the height direction UZ can be seen in Figure 4 and Figure 5.

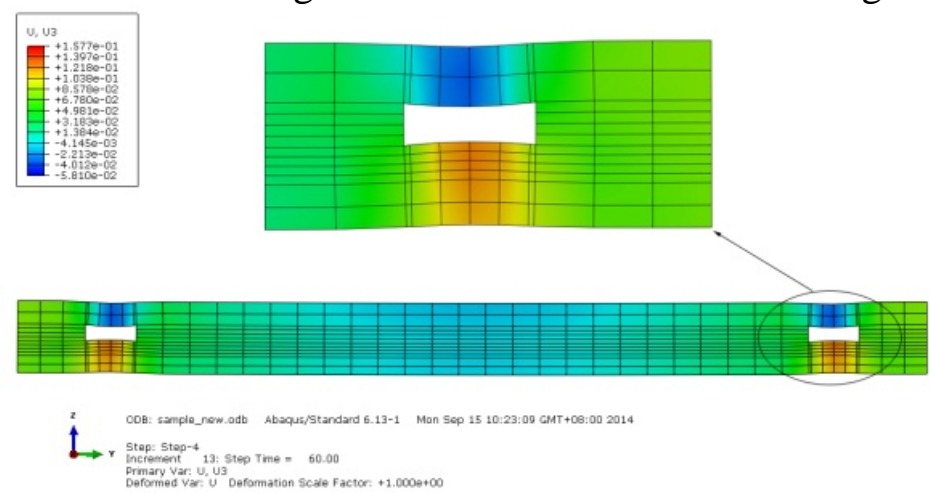

Fig. 4 main channels displacement 


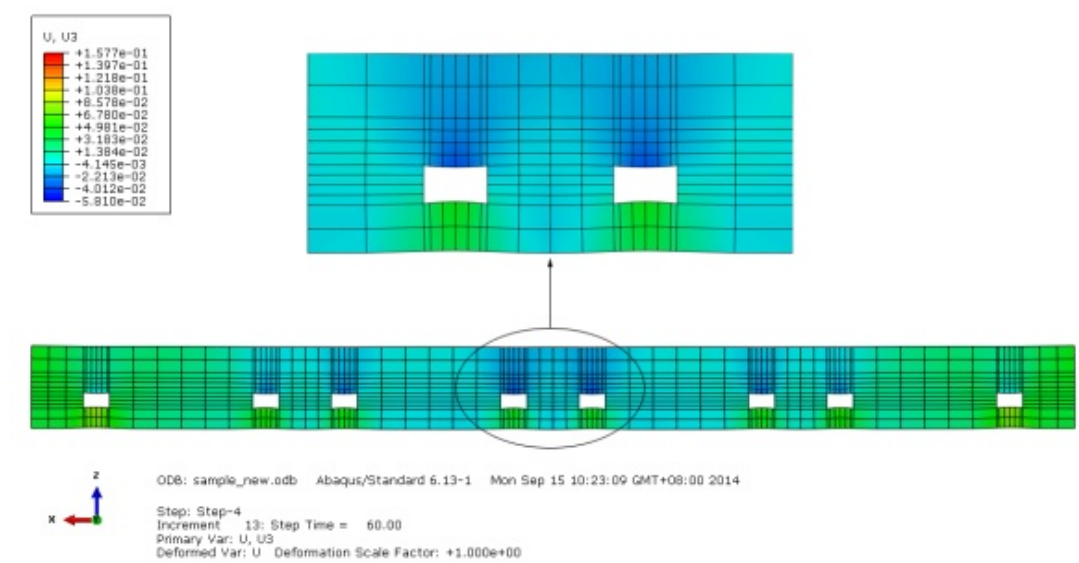

Fig. 5 branch channels displacement

The simulation shows that the micro-channel deformation on the height direction is greater than the deformation on the width direction. This is because that the LTCC microwave module size is larger in $\mathrm{x}-\mathrm{y}$ plane, but smaller in height direction. Under the relative high pressure it is easier to produce deformation on the height direction and the deformation tends to be greater [3]. At the same time, the sag of the main channel with the width $2.2 \mathrm{~mm}$ is more significant than the branch channel with the width $1.0 \mathrm{~mm}$, while the main channel has a large width-height ratio-aspect ratio than branch channel [5].

\subsection{The influence of lamination parameters on micro-channel deformation.}

Study of the influence of lamination parameters on micro-channel deformation, is to change the three lamination parameters pressure, temperature and time value respectively for simulation base on the typical warm water isostatic pressing process. Pressure takes $10 \mathrm{MPa}, 15 \mathrm{MPa}, 20 \mathrm{MPa}$, $25 \mathrm{MPa}$ and $30 \mathrm{MPa}$, temperature takes $50^{\circ} \mathrm{C}, 60^{\circ} \mathrm{C}, 70^{\circ} \mathrm{C}, 80^{\circ} \mathrm{C}$ and $90^{\circ} \mathrm{C}$, and time takes $8 \mathrm{Min}, 10$ Min, 15 Min, 20Min and 25 Min. The main channel cross section deformation on the height direction is obtained under various lamination parameters, the result is shown in Figure 6.

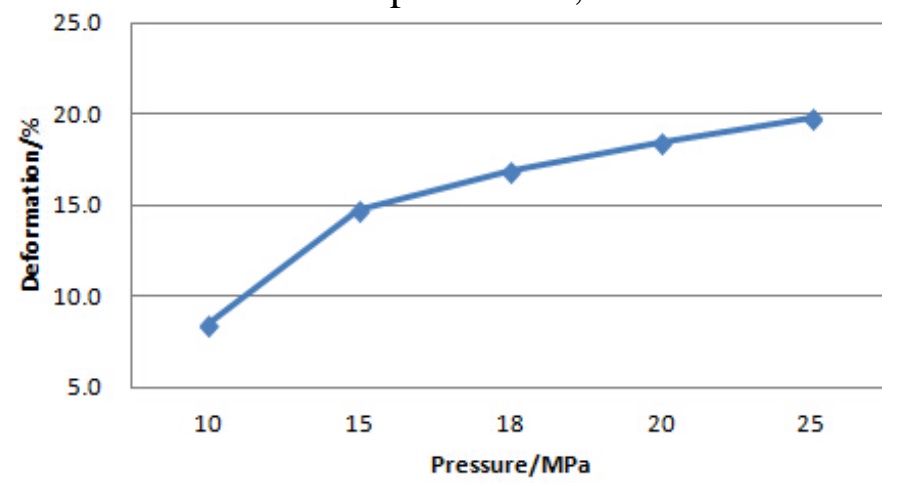

(a) Pressure VS deformation

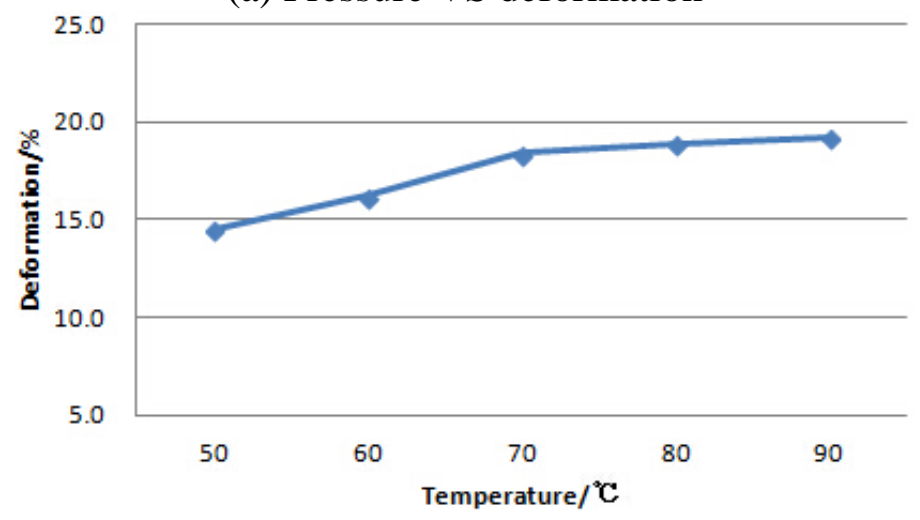

(b) temperature VS deformation 


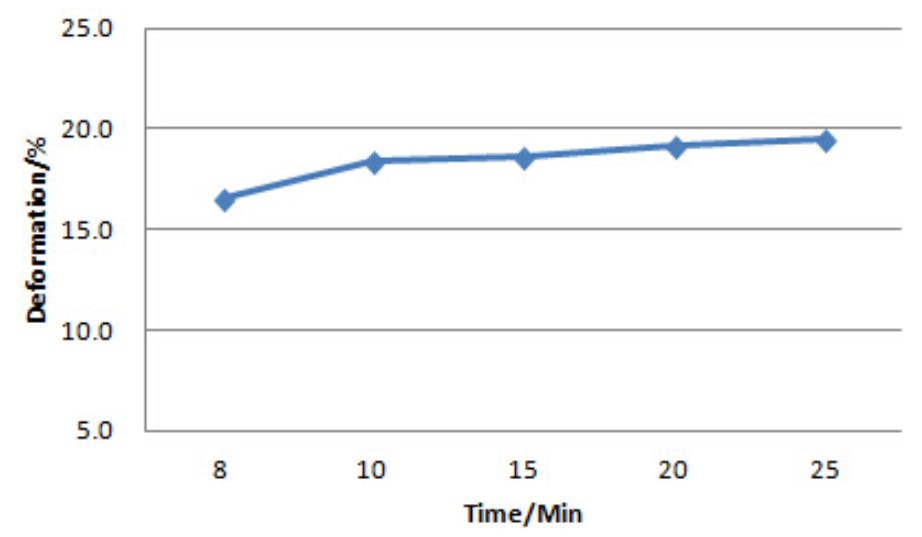

(c) time VS deformation

Fig. 6 the influence of lamination parameters on microchannel deformation

From Fig. 6(a), it can find that the main channel deformation increases with the pressure increasing, and almost close to be a linear relationship. When pressure is $10 \mathrm{MPa}$ the deformation is $8.5 \%$, while pressure is $25 \mathrm{MPa}$ the deformation is $19.8 \%$, the deformation is serious. When the deformation is $15 \%$, pressure is about $16 \mathrm{MPa}$. Hence pressure can be considered to be near $16 \mathrm{MPa}$ in the subsequent parameter optimization. From Fig. 6(b), the main channel deformation also increases with the temperature increasing, but compared with pressure, the trend grows slowly. When temperature is $50^{\circ} \mathrm{C}$ the deformation is $14.5 \%$, while temperature is $90^{\circ} \mathrm{C}$ the deformation is $19.2 \%$. Indicate that when the temperature meets or exceeds the glass transition temperature of the organic binder, the material elastic modulus decreases [3], under a certain pressure, micro-channel suffers great deformation. So a relatively high temperature is not adaptive in the lamination process. From Fig. 6(c), the main channel deformation almost does not change over time. Compare with lamination pressure and temperature, the lamination time does not play a very important role which leads to the channel deformation. It may because that sufficient mechanical relaxation has taken place within the given time for LTCC green tape.

\section{Lamination parameters optimization}

Based on the analysis above, it can find that pressure is the main factor that leads to the deformation of micro-channel, temperature plays a relatively secondary role, and time actually not makes a significant contribution. Hence lower pressure and higher temperature can be chose for LTCC warm water isostatic pressing process. Pressure should be selected from the range of $15 \mathrm{MPa}$ to $20 \mathrm{MPa}$, and temperature should be selected from acthe range of $80^{\circ} \mathrm{C}$ to $90^{\circ} \mathrm{C}$. And time can be much more to insure the LTCC green tapes can join together. However, low pressure is easy to cause de-lamination after sintering. To ensure the quality of LTCC microwave module without delamination, pressure should be close to the upper limit which micro-channel deformation is $15 \%$. Comprehensive consideration, pressure takes $18 \mathrm{Mpa}$, temperature takes $80^{\circ} \mathrm{C}$, time takes 20 Min. To verify the feasibility of this assumption, simulation and sample making are compared, after lamination the main channel displacements are extracted respectively. The main channel crosssection displacements of simulation can be seen in Fig. 7, and the result of sample can be seen in Fig. 8.

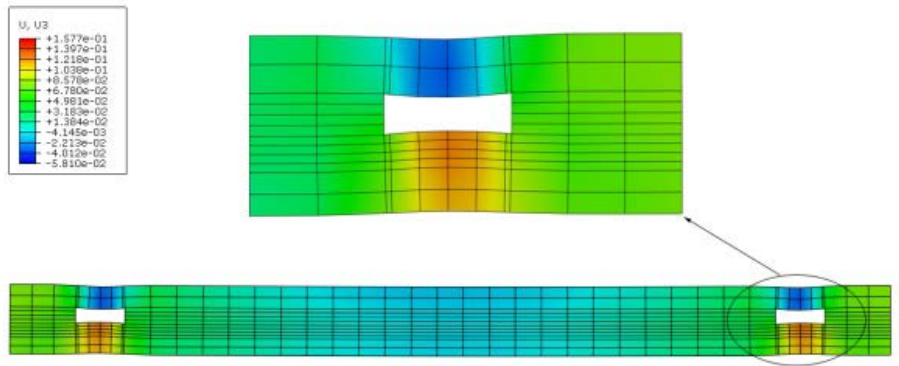

Fig. 7 main channels displacement 


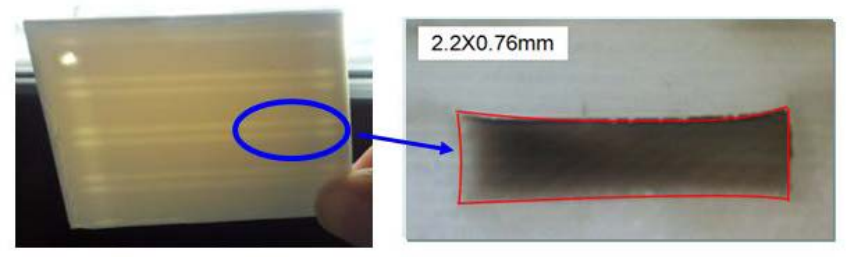

Fig. 8 sample main channels displacement

Results show that the simulation result tallies well with the result of sample making, the crosssections of main channels are mostly consistent. And the micro-channel deformation mainly occurs in the height direction of the channel cross-section. The height deformation is $12.1 \%$ after simulation. The sample is measured with the height of $0.680 \mathrm{~mm}$, the deformation of $10.7 \%$. Compared with the typical lamination process, the deformation reduced $6.3 \%$. Indicate that using the optimized process parameters for the particular LTCC microwave component, micro-channel deformation could be effectively controlled below $15 \%$ after warm water isostatic pressing process.

\section{Conclusions}

In this paper, micro-channel deformation embedded in LTCC microwave component is studied by ABAQUS simulation analysis of warm water isostatic pressing process. Result shows that pressure is the main factor that leads to the deformation of micro-channel. The micro-channel deformation increases with the increasing of pressure and temperature, in contrast, time has a little effect on deformation of micro-channel. SVM plays an important role in supporting the green tape, can effectively reduce the deformation of the micro-channel. To control of micro-channel deformation during isostatic lamination process, lamination pressure must be first taken into account, lower pressure and higher temperature can be chose.

\section{Acknowledgements}

This work was supported by *** basic research project "The Study of *** Multi-energy***” and "Guangxi Key Laboratory of Manufacturing System \& Advanced Manufacturing technology Open Subject Project”.

\section{References}

[1] Zhang,Y. F. et al, Microstructure and mechanical properties of an alumina-glass low temperature co-fired ceramic, Journal of the European Ceramic Society, 29, (6): 1077-1082, 2009.

[2] L.E. Khoong, Y.M. Tan, Y.C. Lam, Study of deformation and porosity evolution of low temperature co-fired ceramic for embedded structures fabrication. Journal of the European Ceramic Society, 29, 2737-2745, 2009.

[3] D. Jurków \& L. Golonka, Low-pressure, thermo-compressive lamination, Journal of the European Ceramic Society, 32, 2431-2441, 2012.

[4] Mu, F. Q. et al, "Process Research of LTCC Substrate with 3D Micro-channel Embedded,” Proc. 10th International conference electron package Tech, pp. 137-140, 2009.

[5] Horváth, G. Hénap, G. Harsányi, Finite Element Modeling of Channel Sag in LTCC, Microelectronics International, 2012, 29, 145-152.

[6] Jones WK, Liu Y, Wang P, Zampino M., "Chemical structural and mechanical properties of LTCC tapes”, Int J Micro Electron Packaging, 23(4), pp.469-73, 2000.

[7] K. Malecha, L.J. Golonka, Microchannel fabrication process in LTCC ceramics. Microelectronics Reliability, 48, 866-871, 2008.

[8] Birol H, Maeder T, Ryser P. Processing of graphite-based sacrificial layer for microfabrication of low temperature co-fired ceramics (LTCC). Sensors and Actuators A, 130-131:560-7, 2006. 
[9] Khoong, L. E., Tan, Y. M., Lam, Y. C. Overview on fabrication of three-dimensional structures in multi-layer ceramic substrate. Journal of the European Ceramic Society, 30 (10): 1973-1987, 2010. 